\title{
DYNAMIC SIMULATION OF THE TEMPORAL RESPONSE OF MICROSTRUCTURE FORMATION IN MAGNETORHEOLOGICAL FLUIDS
}

\author{
H.V. LY, K. ITO, H.T. BANKS \\ Center for Research in Scientific Computation \\ North Carolina State University, Raleigh, NC 27695-8205 \\ M.R. JOLLY \\ Advanced Technologies, Material Division, Lord Corporation \\ 110 Lord Drive, Cary, NC 27511 \\ F. REITICH \\ School of Mathematics, University of Minnesota, Minneapolis, MN 55455 \\ Efficient numerical simulations of microstructure development in magnetorheolog- \\ ical (MR) fluids are conducted. The simulations, which are based upon a fast \\ multipole algorithm, treat the magnetic inclusions as two-dimensional continuum \\ magnetic entities. The development of microstructure is quantified by computing \\ and recording the time evolution of the effective permeability of the composite \\ fluid. Such a principle has been previously exploited for the experimental mea- \\ surements of microstructure development [Jolly, Bender and Mathers, ERMR'97, \\ Yonezawa, Japan 1997]. As was observed experimentally, numerical simulations \\ reveal the evolution of microstructure to be multimodal in nature. Unlike the ex- \\ periments, the numerical simulations afford us the ability to observe the physical \\ mechanisms associated with various modes.
}

\section{Introduction}

The nature of microstructure formation in controllable fluids has been a topic of recent considerable interest. It is generally believed that the field responsive rheological effect is sensitive to the nature of the microstructure ${ }^{1}$. Further, it has been postulated that the time associated with structure formation is an important constituent of the overall time required for the development of stress within controllable fluids ${ }^{2}$. There has been considerable work to understand the nature and temporal behavior of structure formation in electrorheological (ER) fluids through analytical models ${ }^{3,4,5}$ and numerical simulations $s^{6,7}$. Fewer analogous magnetorheological (MR) fluid studies are found in the literature ${ }^{8,9}$.

In a previous paper ${ }^{10}$, we have measured the time scales associated with microstructure development in MR fluids by examining the evolution of fluid permeability (via. polarization measurements) in response to a step change in applied magnetic field. This experimental technique is somewhat analogous to the use of dielectric measurements ${ }^{11,12,13}$ to infer microstructure development in ER fluids in that the dielectric response of an ER fluid is studied in response to an electrical stimulus. These studies of MR fluids indicated that the microstructure response was well-fitted with a bi-exponential function where the two time scales differed by about a factor of five. The first experimental time constant was found to be somewhat consistent with predicted flocculation times based on the computed time

hawaii990823: submitted to World Scientific on August 23, 1999 
of collision of two dipoles in a viscous media ${ }^{3}$. Inconsistency was found in the relationship to volume fraction $\phi$ - the simple theory predicts $t \sim \phi^{-n}$ where $n=5 / 3$, whereas $n \in[2 / 3,4 / 3]$ was observed experimentally.

The notion that microstructure formation occurs at multiple time scales has been observed in the work of others. Halsey and Toor ${ }^{4}$ used some elegant theory to argue that structure formation in ER fluids is a two step process: an initial aggregation to a chain-like structure resulting from electrostatic interaction and a phase separation to a thermodynamic ground state. The latter process is a result of thermally induced fluctuations within the chains and occurs on thermal diffusion time scales. Through numerical simulations that neglected thermal forces, Mohebi and colleagues $^{8}$ showed that structure formation in MR fluids $(\phi=10 \%)$ occurs at two distinct time scales. These time scales correspond to an initial formation of disparate chains, and then a migration of chains into longer and thicker structures. The first process was seen to occur on the millisecond time scale and the second process was observed to be one to three orders of magnitude slower depending upon the sample thickness. Hass ${ }^{7}$, using similar dynamic simulations on ER fluids, demonstrated two initial time scales separated by an order of magnitude which he attributes to, first, pair formation and, second, percolating column growth. The experimental work of Jolly and colleagues ${ }^{10}$ exhibited time scales that are comparable to those found in the latter two simulation studies, but could provide no insight into the mechanisms associated with these time scales.

In this paper, a novel two-dimensional simulation of MR fluid microstructure dynamics is presented. This simulation treats the particle inclusions as twodimensional magnetic entities. The time evolution is considered to be magnetically quasi-static and magnetostatic forces are derived from the solution of (steady) Maxwell's equations, recomputed at each instant in time. For this we use a potential theoretic formulation where the boundary integral equations are solved with a fast multipole method ${ }^{14}$. The simulations are then used to explore the multimodal nature of microstructure development in MR fluids. These results are compared to previously reported experimental results ${ }^{10}$.

\section{Equations of Motion}

In this section, we describe the equations of motion for $M$ permeable circular particles $\left\{\Omega_{k}\right\}_{k=1}^{M}$ in a rectangular container $\Omega \subset \mathbb{R}^{2}$ that is filled with a non-magnetic viscous fluid. The particles are randomly distributed initially and the external magnetic field $\overrightarrow{\mathbf{H}}_{0}$ is applied in the vertical direction. The motion of the kth particle is governed by Newton's second law of motion. Particle interactions include magnetic, hydrodynamic and repelling forces while Brownian and inertial forces are neglected. By performing dimensional analysis and eliminating small-scale terms (for details, see Ly et.al. ${ }^{15}$ ), we obtain

$$
\frac{d \overrightarrow{\mathbf{x}}_{k}}{d t}=\overrightarrow{\mathbf{F}}_{k}^{\text {mag }}+\overrightarrow{\mathbf{F}}_{k}^{\text {rep }} \quad \text { and } \quad \overrightarrow{\mathbf{x}}_{k}^{0}=\overrightarrow{\mathbf{x}}_{k}(0)
$$

where $\overrightarrow{\mathbf{x}}_{k}, t, \overrightarrow{\mathbf{F}}_{k}^{\mathrm{mag}}, \overrightarrow{\mathbf{F}}_{k}^{\mathrm{rep}}$, are dimensionless variables for position, time, magnetic force and repelling force, respectively. 
The spatial scale is $R$, where $R$ the the particle radius and the temporal scale is $\frac{D}{\mu_{0} H_{0}^{2}}$, where $D$ is the Stokes' drag coefficient, $\mu_{0}$ is the permeability for the carrier oil, and $H_{0}$ is the magnitude of the applied field.

For our simulations, we shall assume that both the particles and the container walls are "hard". To approximate this regime, we shall follow the work of Klingenberg et.al. ${ }^{6}$ and propose that a "repelling force" acts on the kth particle as it approaches others or a wall of the container. A simple model for such a force is given, for instance, by

$$
\overrightarrow{\mathbf{F}}_{k}^{\mathrm{rep}}=-\sum_{l=1}^{M} \exp \left(-\beta \frac{\left|d_{k l}\right|-2 R}{2 R}\right) \overrightarrow{\mathbf{r}}_{k l}-\exp \left(-\beta \frac{\left|d_{k}^{\text {wall }}\right|-2 R}{2 R}\right) \overrightarrow{\mathbf{n}}_{k},
$$

where $\overrightarrow{\mathbf{r}}_{k l}=\frac{\overrightarrow{\mathbf{x}}_{l}-\overrightarrow{\mathbf{x}}_{k}}{\left|\overrightarrow{\mathbf{x}}_{l}-\overrightarrow{\mathbf{x}}_{k}\right|}, d_{k l}=\operatorname{dist}\left(\Omega_{k}, \Omega_{l}\right)$, and $\beta>0$ is the repelling parameter. The wall repelling force uses $\overrightarrow{\mathbf{n}}_{k}$, an outward unit normal vector at a point $p$ on the boundary of the container $\Omega$ where $p$ is nearest to $\overrightarrow{\mathbf{x}}_{k}$ on $\partial \Omega$ and $\left|d_{k}^{\text {wall }}\right|=\operatorname{dist}\left(\Omega_{k}, \Omega\right)$.

The magnetic force on $\Omega_{k}$ can be calculated from the local field $\overrightarrow{\mathbf{H}}$ with the aid of the Maxwell stress tensor $\sigma^{\mathrm{Max}}=\mu_{0}\left[\overrightarrow{\mathbf{H}} \overrightarrow{\mathbf{H}}^{\mathcal{T}}-\frac{1}{2}|\overrightarrow{\mathbf{H}}|^{2} \delta\right],(\delta=$ unit tensor $)$ as

$$
\overrightarrow{\mathbf{F}}_{k}^{\operatorname{mag}}=\frac{1}{2 \pi \mu_{0} H_{0}^{2} R} \int_{\partial \Omega_{k}} \sigma^{\operatorname{Max}} \cdot \overrightarrow{\mathbf{n}}_{k} d S,
$$

where $\overrightarrow{\mathbf{n}}_{k}$ is the unit normal vector on $\partial \Omega_{k}$. An accurate estimate for the magnetic force in (3) demands the continuous knowledge of the local magnetic field $\overrightarrow{\mathbf{H}}$, so that Maxwell's equations must be resolved at each instant in time. In our simulation, we assume there are no free currents in the domain, so that the local magnetostatic field $\overrightarrow{\mathbf{H}}$ can be written in terms of a scalar potential $\Phi$,

$$
\overrightarrow{\mathbf{H}}=-\nabla \Phi .
$$

Moreover, $\Phi$ is the solution of the Laplace's equation

$$
\nabla \cdot(\mu \nabla \Phi)=0
$$

with highly oscillatory coefficients

$$
\mu= \begin{cases}\mu_{k} & \text { in the kth particle, } \\ \mu_{0} & \text { in the carrier oil. }\end{cases}
$$

For our simulation, we assume $\mu_{k} \sim 2000 \mu_{0}$. Along with equation (5) we require the continuity of the magnetic potential $\Phi$ and of the normal component of $\overrightarrow{\mathbf{B}}$. That is, for any $k=1,2, \ldots, M$,

$$
\begin{gathered}
\lim _{\substack{p \rightarrow \partial \Omega_{k} \\
p \in \Omega_{k}}} \Phi(p)=\lim _{\substack{p \rightarrow \partial \Omega_{k} \\
p \in \Omega_{k}^{c}}} \Phi(p), \\
\lim _{\substack{p \rightarrow \partial \Omega_{k} \\
p \in \Omega_{k}}} \mu_{k} \frac{\partial \Phi}{\partial \vec{n}_{k, p}}(p)=\lim _{\substack{p \rightarrow \partial \Omega_{k} \\
p \in \Omega_{k}^{c}}} \mu_{0} \frac{\partial \Phi}{\partial \vec{n}_{k, p}}(p),
\end{gathered}
$$

where $\vec{n}_{k, p}$ is an outward unit normal vector at $p \in \partial \Omega_{k}$ and $\Omega_{k}^{\mathrm{c}}=\Omega \backslash \bar{\Omega}_{k}$ is the complement of $\Omega_{k}$. 


\section{$3 \quad$ Integral Equation Formulation and Boundary Element Discretization}

Although the coefficients of the Laplace's equation (5) are rapidly changing in space, they do remain constant in each $\Omega_{k}$. Thus the overall potential can be derived from appropriate charge densities supported on the boundaries of the particles. These densities satisfy certain integral equations which are, in principle, amenable to solution by finite (boundary) element approximation. As we discussed in detail elsewhere ${ }^{15}$, the difficulties associated with the high computational cost of classical boundary element approximation for this kind of problem can, in fact, be overcome through the implementation of the Fast Multipole Method ${ }^{14}$.

To derive the integral equations, let us denote by $\partial \Omega_{0}$ the boundary of the domain $\Omega$. We also denote by $\Omega_{0}$ the fluid region $\Omega \backslash\left\{\cup_{k=1}^{M} \Omega_{k}\right\}$ and impose the following Neumann boundary condition on $\partial \Omega_{0}$

$$
\left.\frac{\partial \Phi}{\partial \vec{n}}\right|_{\partial \Omega_{0}}=g
$$

To guarantee the solvability and uniqueness of the solution of equations (5)-(9), we make the following requirements on $g$ and $\Phi$; namely,

$$
\int_{\partial \Omega_{0}} g d s=0 \quad \text { and } \quad \int_{\Omega} \Phi d \overrightarrow{\mathbf{x}}=0 .
$$

A potential $\Phi$ satisfying (5)-(9) can be represented by single-layer potentials ${ }^{16}$ in the form

$$
\Phi(p)=\sum_{j=0}^{M} \int_{\partial \Omega_{j}} G(p, q) \xi_{j}(q) d s(q), \quad p \in \Omega .
$$

Here, $G(p, q)=\frac{1}{2 \pi} \log |p-q|$ is the fundamental solution of the Laplace's equation in $\mathbb{R}^{2}$. The functions $\xi_{j}$ 's on $\left\{\partial \Omega_{j}\right\}_{j=0}^{M}$ are appropriate (unknown) surface densities. Note that the potential $\Phi$ in (10) automatically satisfies $\Delta \Phi=0$ on $\Omega_{k}$ for $k=$ $0,1, \ldots, M$, and the continuity condition ( 7$)$ at the interfaces. In addition, using the jump relations of potential theory ${ }^{16}$, we obtain from equations (8) and (9) the following system of Fredholm equations of the second kind,

$$
\begin{aligned}
\xi_{0}(p)-2 \sum_{j=0}^{M} \int_{\partial \Omega_{j}} \frac{\partial}{\partial \vec{n}_{p}} G(p, q) \xi_{j}(q) d s(q) & =-2 g(p), \\
\xi_{k}(p)-2 \lambda_{k} \sum_{j=0}^{M} \int_{\partial \Omega_{j}} \frac{\partial}{\partial \vec{n}_{p}} G(p, q) \xi_{j}(q) d s(q) & =0,
\end{aligned}
$$

where $\lambda_{k}=\frac{\mu_{k}-\mu_{0}}{\mu_{k}+\mu_{0}}$ and equations (11)-(12) hold for $p \in \partial \Omega_{0}$ and $\left\{\partial \Omega_{k}\right\}_{k=1}^{M}$, respectively. Equations (11)-(12) are then discretized and solved with the help of the fast multipole method ${ }^{14}$. A similar approach with details, implemented for MR applications, can be found in our earlier work ${ }^{15}$, where the Dirichlet boundary conditions were assumed at the exterior boundary and the resulting formulations involved both the single- and double-layer integral potentials. We also remark here

hawaii990823: submitted to World Scientific on August 23, 1999 
that because our the potential formulation $\Phi$ in (10) consists of only the single formulation, numerical implementation is more efficient and integral singularities are removable. Numerical results are discussed in the next section.

\section{Numerical Results and Discussion}
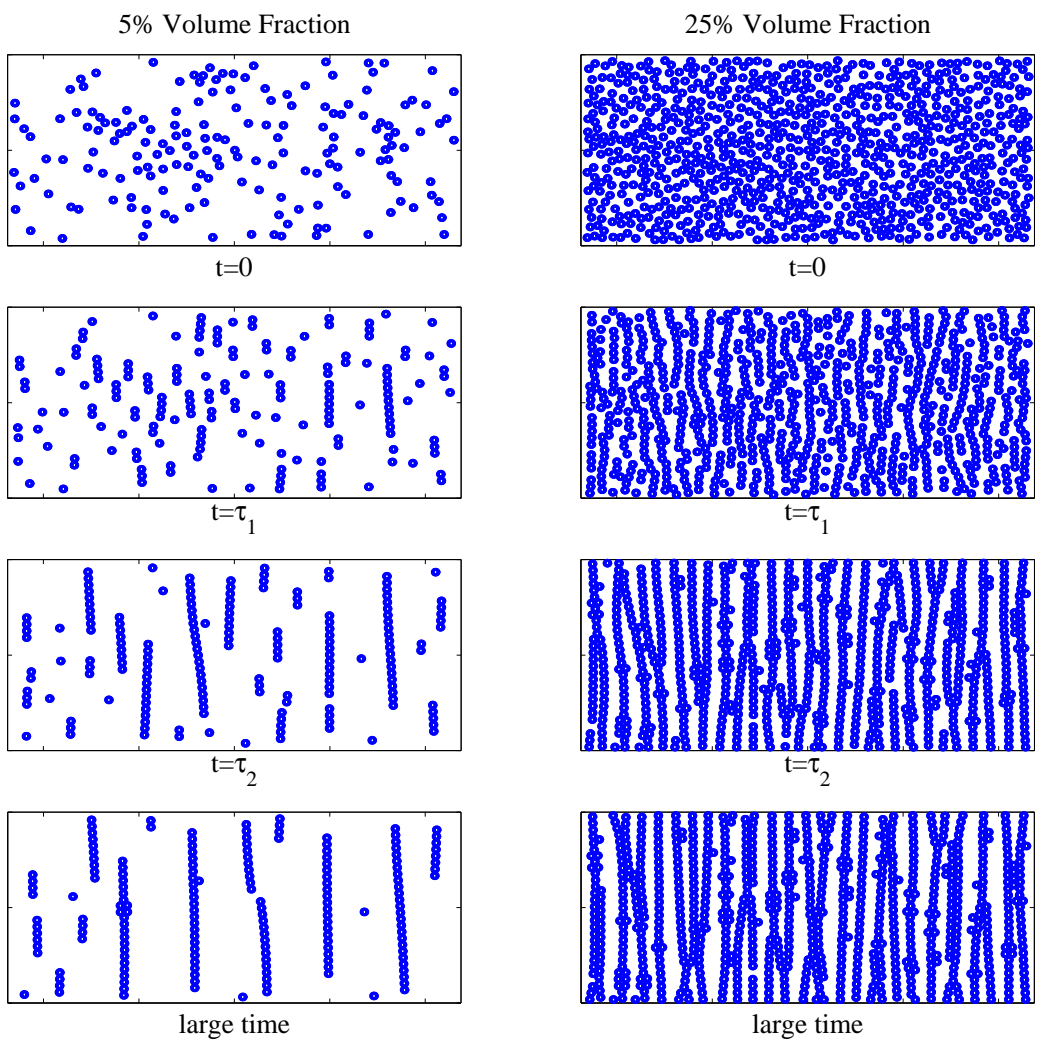

Figure 1. Microstructure formation for $5 \%$ and $25 \%$ volume-fraction samples in four stages ( $t$ =initial, first time scale $\left(\tau_{1}\right)$, second time scale $\left(\tau_{2}\right)$, and large time).

Simulations were conducted on six different volume fractions ranging from $5 \%$ to $30 \%$. The external field is applied in the vertical direction. Simulation results for two volume fractions responding to a step field input are shown in Figure 1. Discrete times within the simulation are shown from the initial configuration to the near steady state microstructure. An issue in such dynamic simulations involves the means by which the state of the microstructure is quantified. Researchers have used quantities related to nearest neighbor inter-particle distances and spatial correlation functions to monitor the state of a microstructure ${ }^{17,5}$. Others have used mean square particle displacement ${ }^{6,7}$. In a manner analogous to a previously reported experimental technique ${ }^{10}$, we will use the composite magnetic permeability to quan- 


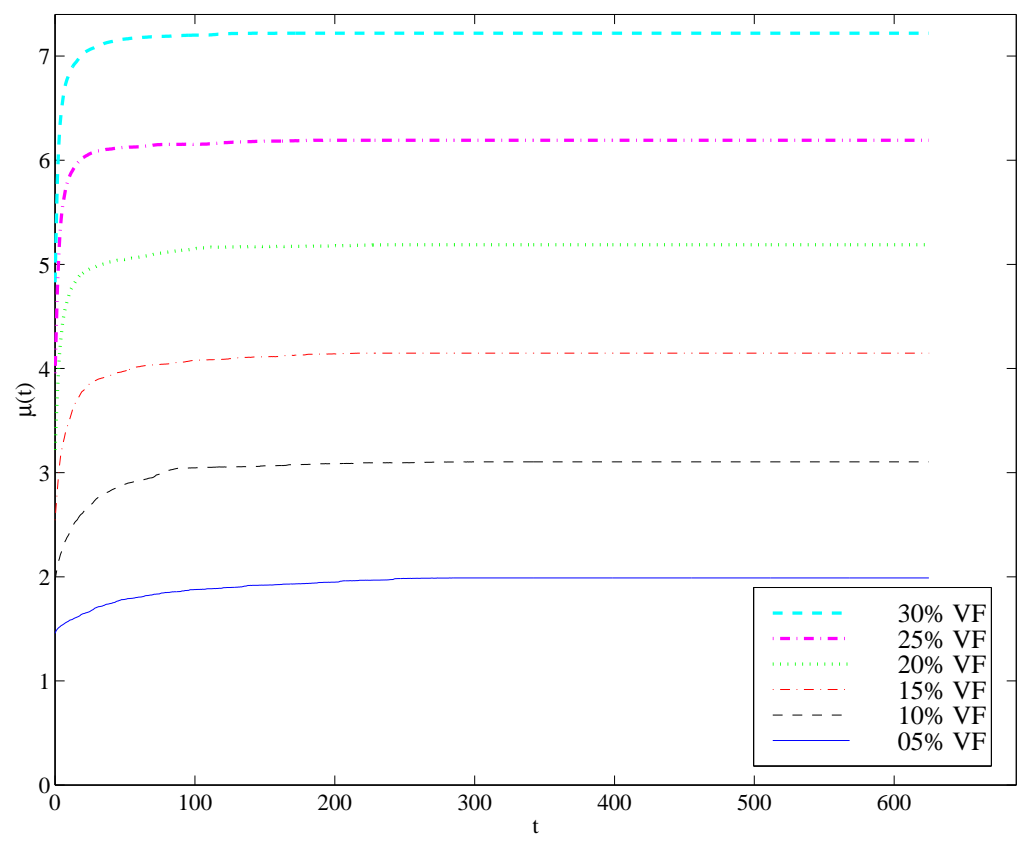

Figure 2. Simulated effective permeability $\bar{\mu}(t)$ as a function of non-dimensional time $t$ for six different volume fractions.

tify the evolution of microstructure. This method exploits the fact that a percolated microstructure is significantly more permeable than a randomly mixed microstructure. Some theoretical limits of this behavior are discussed in Simon et.al. ${ }^{18}$. It was reported by Ly et.al. ${ }^{15}$, that the evolution of permeability in microstructure formation is closely related to the mean particle velocity. During the simulation process, we compute the effective permeability $\bar{\mu}(t)$, which represents the overall response for the microstructure formation. The definition and the formulation for the effective permeability, which is based on the theory of homogenization ${ }^{19}$, are derived in Ly et.al. ${ }^{20}$. Figure 2 shows the permeability response of the six samples to a step input in magnetic field. It should be pointed out that the simulations are displayed in dimensionless times and dimensional times can be obtained by multiplying the dimensionless times with the time scale described earlier in Section 2. As expected, both the initial and the steady state permeabilities are linearly related to the volume fraction, $\phi$.

As was found experimentally, the permeability response is well-fitted with a bi-exponential function. These fits are also applied to the permeabilities shown in Figure 2 and the resultant time constants and coefficients are presented in Table 1. Microstructure frames corresponding to the two time constants $\left(\tau_{1}\right.$ and $\left.\tau_{2}\right)$ are shown in Fig. 1. From these frames, it is evident that the first mode corresponds to particle pair and short chain formation and the second mode corresponds to coalescence of the short chains into longer percolating chain structures. Similar

hawaii990823: submitted to World Scientific on August 23, 1999 
observations from simulations of ER and MR fluids have been made by others including $\mathrm{Hass}^{7}$ and Mohebi et.al. ${ }^{8}$. The two time constants are separated by a factor of 5 to 10. An additional observation can be made in noting that magnetic energy is proportional to permeability for linear materials. In particular, it can be seen that about $65-70 \%$ of the microstructures magnetic potential energy is stored upon initial application of the field. The balance of the stored magnetic energy occurs during the microstructure formation. For high volume fraction fluids, over $90 \%$ of the energy is stored within the first time constant dropping to below $70 \%$ as volume fraction decreases.

\begin{tabular}{|c|c|c|c|c|c|}
\hline \multicolumn{6}{|c|}{ Bi-Exponential Fit } \\
$\bar{\mu}(t)=A *\left(1-e^{t / \tau_{1}}\right)+B *\left(1-e^{t / \tau_{2}}\right)+\mu(0)$ \\
\hline$\phi$ & $\tau_{1}$ & $\tau_{2}$ & $A$ & $B$ & $\mu(0)$ \\
\hline $5 \%$ & 16.2 & 88.8 & 0.170 & 0.368 & 1.46 \\
$10 \%$ & 8.81 & 43.8 & 0.467 & 0.667 & 1.97 \\
$15 \%$ & 5.87 & 46.1 & 1.090 & 0.517 & 2.54 \\
$20 \%$ & 3.93 & 44.2 & 1.550 & 0.418 & 3.22 \\
$25 \%$ & 3.35 & 38.6 & 1.960 & 0.281 & 3.95 \\
$30 \%$ & 3.12 & 29.9 & 2.030 & 0.359 & 4.83 \\
\hline
\end{tabular}

Table 1. Effective permeability and bi-exponential fit.

Figure 3 depicts the first time constant as a function of volume fraction $\phi$. Simulation results as well as experimental results from Jolly et.al. ${ }^{10}$ are presented where the simulation results have been dimensionalized to correspond with the experimental conditions. As shown in several references ${ }^{3,21,10}$, simple theory suggests that the relationship between microstructure formation time and volume fraction should follow a power law behavior with a power index of $n=-5 / 3$. In particular, it has been argued that the time for pair formation is proportional to

$$
t c \sim \frac{\eta \mu_{0}}{J_{p}^{2}}\left[\left(\frac{\pi}{6 \phi}\right)^{5 / 3}-1\right],
$$

where $J_{p}$ is the particle polarization. Our simulation results have indicated that the first time constant is inversely proportional to volume fraction $(n=-1)$ and experimental results have yielded power indices between $-4 / 3$ and $-2 / 3$. The disparity of this behavior with the simple theory is not surprising since the theory is based on two isolated particles (magnetically modeled as dipoles) in a viscous medium. We further note that our simulations do not account for the magnetic nonlinearity of the particle material, which may contribute to the range of power law indices in the experimental data. If it is assumed that $J_{p}=B_{i} / \phi$, where $B_{i}$ is the intrinsic induction of the composite, then $B_{i}$ can be substituted into Eq. (13) and the aforementioned power law index becomes $n+2$ (hence, $n+2=1 / 3$ for theory, $n+2=1$ for simulation and $2 / 3<(n+2)<4 / 3$ for experiments $)$. Figure 4 contains previously reported ${ }^{10}$ experimental time constants and scaled simulation time constants as a function of $\eta \phi / B_{i}^{2}$. It can be seen that the time constants 
rise linearly with this function. The numerical results are shifted with respect to experimental results. This may be the result of structured error in the experimental measurement of $B_{i}$.

\section{Conclusions}

A numerical algorithm has been developed to accurately study both the microstructure and the permeability with the aid of the fast multipole method. The results have been quantified with the two time scales for structure formation $\left(\tau_{1}\right.$ : particle pairing and short-chain formation; $\tau_{2}$ : coalescence of short chains into longer percolating chain structures). Our investigation reveals that the first time scale, $\tau_{1}$, is inversely proportional to the particle volume fraction - a result that is in reasonable agreement with previous experimental results ${ }^{10}$. It is anticipated that the use of permeability (or polarization) measurements to monitor the state of microstructure in MR fluids will be particularly useful in shear. Other means of quantifying microstructure, especially those involving monitoring mean particle motion, may fall short in shear environment. Permeability measurements from both particle simulation and experiments should be capable of resolving microstructure formation and the shear-induced microstructure degradation. Correlation between such measurements and the MR response will be a topic of future research.

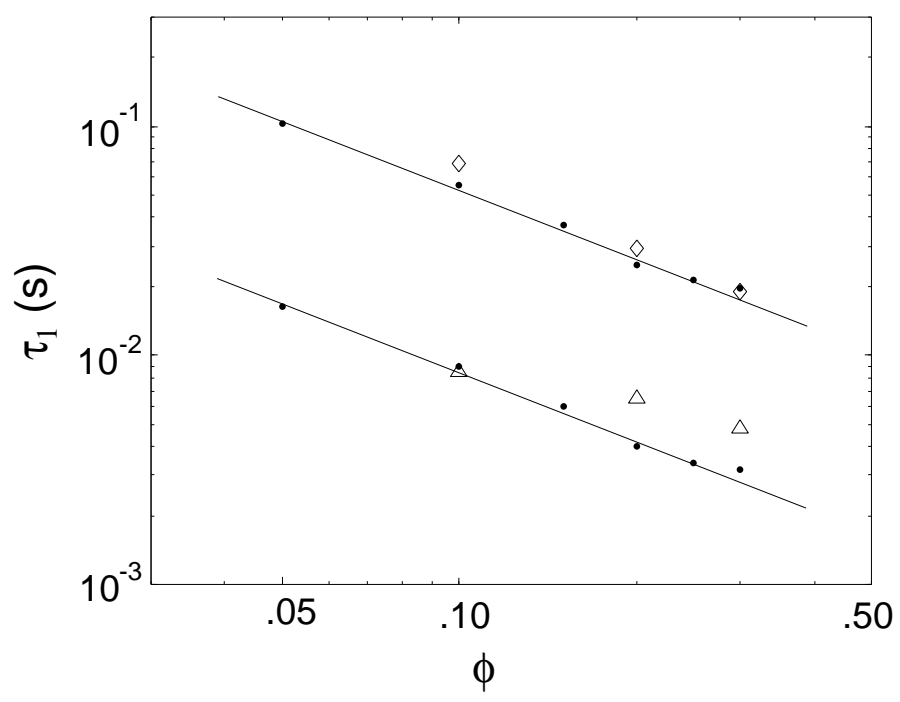

Figure 3. The first time constant as a function of volume fraction: (triangles)-experimental results for $H=17.5 \mathrm{kA} / \mathrm{m}$ and $\eta=0.13 \mathrm{~Pa}-\mathrm{s}$; (diamonds)-experimental results for $H=7 \mathrm{kA} / \mathrm{m}$ and $\eta=0.13 \mathrm{~Pa}$-s. The small circles are simulation results that have been dimensionalized to match the corresponding experimental results. Inverse linear fits are also shown. Experimental results are from Jolly et.al ${ }^{10}$.

hawaii990823: submitted to World Scientific on August 23, 1999 


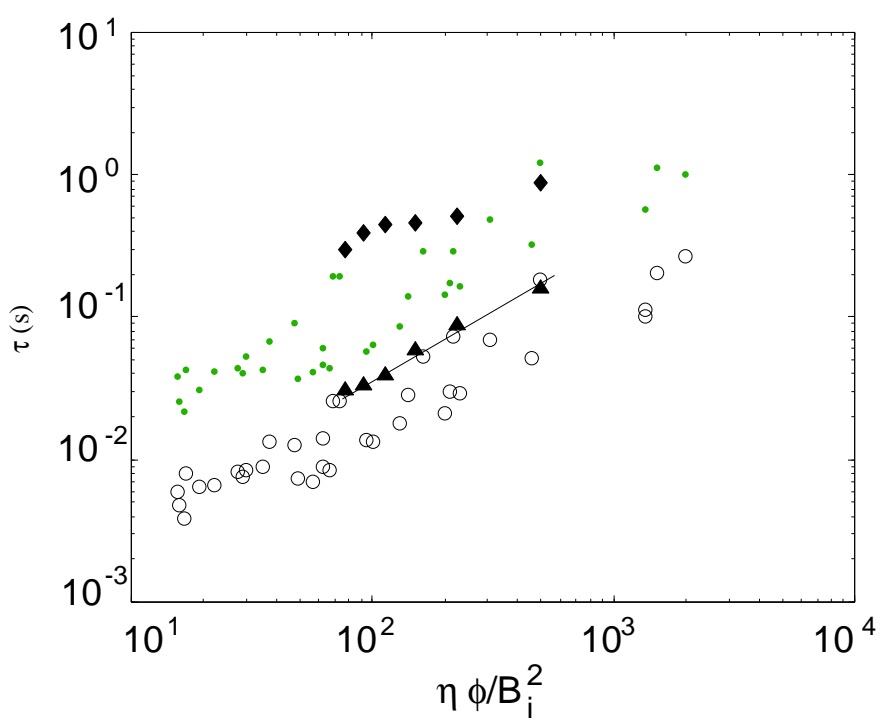

Figure 4. Microstructural response times as a function of their assumed theoretical dependency. Triangles and diamonds are the first and second dimensionalized time constants from simulation. Circles and dots are the first and second experimentally measured time constants ${ }^{10}$.

\section{Acknowledgments}

This work was supported jointly by a National Science Foundation Industrial PostDoctoral Grant (No. DMS-9704963) and by the Lord Corporation through funds to the Center for Research in Scientific Computation at North Carolina State University. The research in this project was done when Hung V. Ly was a National Science Foundation Industrial Post-Doctoral Fellow at the Center for Research in Scientific Computation- North Carolina State University. His current address is Department of Mathematics, California State University, Fullerton, CA 92834-6850. F. Reitich gratefully acknowledges support from AFOSR through grant No. F49620-95-1-0113 and from NSF through grant No. DMS-9622555. H.T. Banks was supported in part by AFOSR under grants F49620-95-1-0236 and F49620-98-1-0180.

\section{References}

1. M. Parthasarathy and D.J. Klingenberg, Proc. XIIth Int. Congr. on Rheology, ed. A. Ait-Kadi, J.M. Dealy, D.F. James and M.C. Williams (Can. Rheology Group) p. $311,(1996)$.

2. J.M. Ginder and S.L. Ceccio, J. Rheol. 39, 211-234 (1995).

3. M. Shapiro, A.L. Shalom and I.J. Lin J. Appl. Phys. 58, N2:1028-1031 (1985).

4. T.C. Halsey and W. Toor, Phys. Rev. Lett. 65, N22:2820-2823 (1990).

5. A.P. Gast and C.F. Zukoski, Adv. Colloid Interface Sci. 30, 153-202 (1989).

6. D.J. Klingenberg, F. van Swol, C.F. Zukoski, J. Chem. Phys. 91, N12:7888 (1989).

7. K.C. Hass, Phys. Rev. E 47, N5:3362-3373 (1993). 
8. M. Mohebi, N. Jamasbi and J. Liu, Phys. Rev. E 54, N5:5407-5413 (1996).

9. C.S. Yeh and K.C. Chen, Continuum Mech. Thermodyn. 9, 273-291 (1997).

10. M. R. Jolly, J.W. Bender and R.T. Mathers, Proc. Int. Conf. on ER Fluids, MR Suspensions and Appl., Yonezawa, JP, July 22-25 , 470-477 (1997).

11. K.D. Weiss and J.D. Carlson, in Electrorheological Fluids, ed. R. Tao (World Scientific, Singapore) p.264, (1992).

12. K.M. Blackwood, H. Block, P. Rattray, G. Tsangaris and D.N. Vorobiev, in Electrorheological Fluids, ed. R. Tao and G.D. Roy (World Scientific, Singapore) p.3 , (1994).

13. D. Adolf and T. Garino, Langmuir 11, 307-312 (1995).

14. L. Greengard and V. Rokhlin, J. Comput. Phys. 73, N2:325 (1987).

15. H.V. Ly, F. Reitich, M. Jolly, H.T. Banks, and K. Ito, J. Comput. Phys. to appear, (1999).

16. R. Kress, Linear Integral Equations, Springer-Verlag, Berlin Heidelberg (1989).

17. J. Liu, E.M. Lawrence, A. Wu, M.L. Ivey, G.A. Flores, K. Javier, J. Bibette, and J. Richard, Phys. Rev. Lett. 74, N14:2828-2831 (1995).

18. T. Simon, F. Reitich, M. R. Jolly, K. Ito, and H. T. Banks, CRSC TR-98-35, North Carolina State University (1998), Journal of Intelligent Material Systems and Structures (submitted), (1998).

19. V. V. Jikov, S. M. Kozlov, and O. A. Oleinik, Homogenization of Differential Operators and Integral Functionals, Springer-Verlag, New York, (1994).

20. H.V. Ly, F. Reitich, M. Jolly, H.T. Banks, and K. Ito, In Preparation, (1999).

21. J.M. Ginder, in Encycl. Appl. Phys. (VCH Publ. Inc.) 16, 487-503 (1996). 